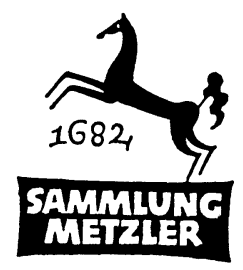

REALIEN ZUR LITERATUR

ABT. F.:

WECHSELBEZIEHUNGEN 


\section{Deutsche und europäische Romantik}




\section{Stuart Atkins in Verehrung}

CIP-Kurztitelaufnahme der Deutschen Bibliothek

\section{Hoffmeister, Gerhart}

Deutsche und europäische Romantik. - 1. Aufl. Stuttgart: Metzler, 1978

(Sammlung Metzler; M 170: Abt. F, Wechselbeziehungen)

ISBN 978-3-476-10170-9

ISBN 978-3-476-10170-9

ISBN 978-3-476-03876-0 (eBook)

DOI 10.1007/978-3-476-03876-0

\section{170}

C) Springer-Verlag GmbH Deutschland 1978

Ursprünglich erschienen bei J. B. Metzlersche Verlagsbuchhandlung und Carl Ernst Poeschel Verlag GmbH in Stuttgart 1978 


\section{INHALT}

Vorbemerkung .............. VII

Abkürzungsverzeichnis ............. IX

A. Einletrung . . . . . . . . . . . . . . 1

1. Zur Wort- und Begriffsgeschichte ........ 1

2. Romantik und Politik . . . . . . . . . . . 12

a) Daten zum Verständnis der historischen Situation . . 13

b) Zur theoretischen Verflechtung . . . . . . . 17

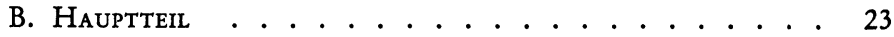

I. Die historische Perspektive . . . . . . . . . . 23

1. .Vorromantik , . . . . . . . . . . 23

2. Die deutsche Romantik. . . . . . . . . 30

3. Die englische Romantik . . . . . . . . 38

4. Die französische Romantik . . . . . . . . . 44

5. Die italienische Romantik. . . . . . . . 50

6. Die spanische Romantik . . . . . . . . . 54

7. Polnische und russische Romantik . . . . . 58

II. Literarische Wechselbeziehungen . . . . . . 63

1. Deutsche und französische Romantik . . . . . . 63

2. Deutsche Romantik und die spanisch-italienische Literatur . . . . . . . . . . . . . . . 68

3. Deutsche und englische Romantik ... . . . 75

Exkurs: Deutsche Romantik in den Vereinigten Staaten

4. Slawische und deutsche Romantik . . . . . . 85

a) Polnisch-deutsche Literaturbeziehungen in der Romantik . . . . . . . . . . . 87

b) Russische und deutsche Romantik . . . . . . . 88

5. Das Bild des Auslandes von der deutschen Romantik 91

6. Der europäische Wirkungsbereich der Romantik . . 94

III. Wesenszüge der europäischen Romantik . . . . . . . 100

1. Asthetik und Poetik der Romantik . . . . . . . . 100

a) Deutscher Idealismus . . . . . . . . . 100

b) Dichter und Dichtkunst . . . . . . . . 103

c) Nachahmung und Schöpfung . . . . . . . . 105

d) Gattungstheorie und -praxis . . . . . . . . 109

e) Stil und Ironie . . . . . . . . . . . 112

2. Weltliteratur . . . . . . . . . 122

a) Mythologie . . . . . . . . . . . . 124 
b) Griechenbild . . . . . . . . . . . . 127

c) Mittelalterauffassung . . . . . . . . . . . 128

d) Exotik . . . . . . . . . . . . . 132

3. Themen, Motive und Gestalten . . . . . . . 137

a) Kampf gegen die Aufklärung. . . . . . . . . 138

b) Der romantische Held . . . . . . . . . . 141

c) Natur und Mensch (Nachtseiten der Natur) . . 161

d) Christliche und satanische Romantik . . . . 166

4. Der romantische Dichter und die Gesellschaft . . 175

a) Isolation und Exil . . . . . . . . . 176

b) Rebellische Dichter, engagierte Literatur . . . 176

c) Die reaktionäre Haltung . . . . . . . . . 178

d) Philisterkritik . . . . . . . . . . . . 179

e) Der literarische Frauensalon . . . . . . . 180

IV. Tradition und Erbe der Romantik . . . . . . . . 184

1. Die Grundlagen der Moderne . . . . . . . . 184

a) Romantik und die politischen Ideologien . . . 184

b) Psychologie (Freud) . . . . . . . . . . . . 185

c) Philosophie (Schopenhauer und Nietzsche) . . 186

d) Wagner . . . . . . . . . . . . 187

2. Literaturperioden . . . . . . . . . . . 188

a) Realistische Romantik . . . . . . . . . 188

b) Dekadenz . . . . . . . . . . . . . . . . 189

c) Expressionismus und Popszene . . . . . . . 192

3. Romantische Aspekte der modernen Literatur .. 193

V. Personenregister . . . . . . . . . . . . . 201 


\section{VORBEMERKUNG}

Diese Arbeit entsprang dem langgehegten Wunsch des Verfassers, aus dem fruchtbaren Chaos der äußerst komplexen Literaturbewegung "Romantik « und der Vielfalt der Forschungsmeinungen $\mathrm{zu}$ einer gewissen Übersicht $\mathrm{zu}$ gelangen. Aus den Unterrichtsbedingungen an den amerikanischen Universitäten ergab sich der komparatistische Ansatz, der eine Darstellung der deutschen Romantik erfordert, ohne sie aus dem europäischen Kontext herauszulösen. $\mathrm{Da}$ die deutsche Romantik die Nachbarliteraturen vielfach entscheidend beeinflußt hat, sollte ihre zentrale Bedeutung auch unter Beachtung des europäischen Standpunktes genügend zur Geltung kommen. Zudem konnte es für einen deutschgebürtigen Autor in kalifornischer Distanz ohnehin keine andere Lösung geben, als aus deutscher Perspektive über die europäische Romantik zu schreiben.

Angesichts der beschränkten Seitenzahl, die für einen Band in dieser Serie zur Verfügung stand, mußte sich der Verfasser auf eine komprimierte Darlegung grundsätzlicher Sachverhalte, Erkenntnisse und Zusammenhänge konzentrieren. So bedauerlich es sein mag, mußte daher auf eine detailliertere Bibliographie und ein Eingehen etwa auf weitere europäische Literaturen z. Zt. der Romantik (in Skandinavien, Portugal, im Balkan etc.) verzichtet werden.

Ein Wort des Dankes möge diese Vorbemerkung beschließen. $\mathrm{Zu}$ einem Thema von einer derartigen Spannweite sind die Bausteine seit Jahrzehnten gelegt worden: Die Einweihung in den Problemkreis verdankt der Verfasser seinem Vater, Johannes Hoffmeister, dessen Vorlesungsmanuskripte er einsehen konnte, den Ausbau seiner Anschauungen den Romantik-Vorlesungen und -Seminaren von Richard Alewyn, Walther Rehm und Benno von Wiese in Bonn und Freiburg, die Überprüfung mancher Thesen seinen eigenen Vorlesungen sowie den Reaktionen seiner Studenten in Milwaukee, Detroit und Santa Barbara, die kritische Durchsicht des Manuskriptes seinen Kollegen Siegfried Mews, Chapel Hill, Stuart Atkins und Rolf Linn, University of California.

Die Verpflichtung gegenüber der Sekundärliteratur kommt in zahllosen Zitaten zum Ausdruck. Um mit einem hier zutreffenden Worte Goethes zu Eckermann zu enden:

"Was können wir dann unser Eigenes nennen, als die Energie, die Kraft, das Wollen! Wenn ich sagen könnte, was ich alles 
großen Vorgängern und Mitlebenden schuldig geworden bin, so bliebe nicht viel übrig« (12. V. 1825).

University of California

G. H.

Santa Barbara 


\section{ABKüRzUngen}

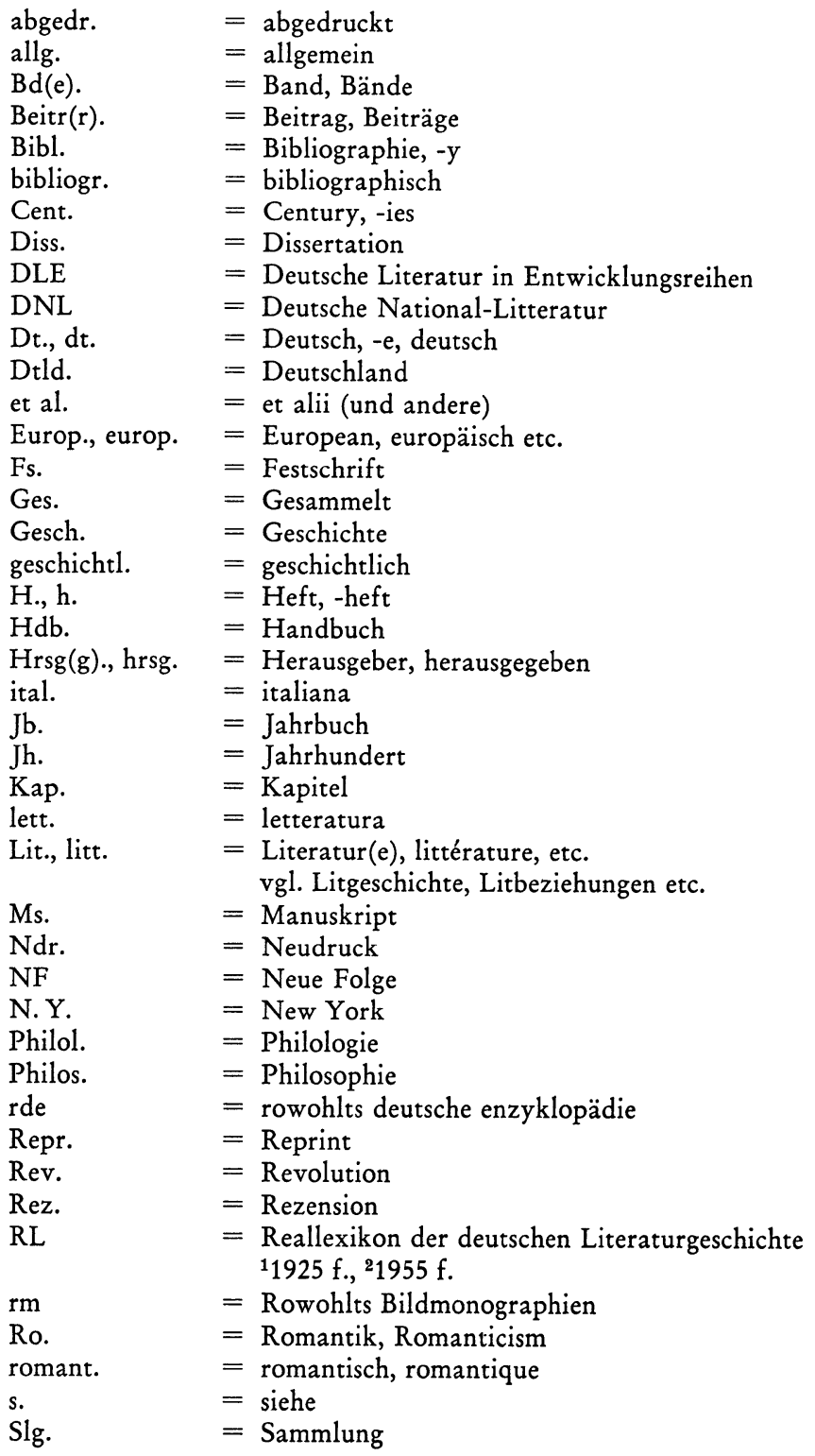




$\begin{array}{ll}\text { Str. } & =\text { Strophe } \\ \text { S. W. } & =\text { Sämtliche Werke } \\ \text { Ubers., übers. } & =\text { Ubersetzung, übersetzt } \\ \text { Unters(s). } & =\text { Untersuchung, -en } \\ \text { U.P. } & =\text { University Press } \\ \text { UTB } & =\text { Universitäts Taschenbuch-Verlag } \\ \text { TEAS } & =\text { Twayne's English Authors Series } \\ \text { TWAS } & =\text { Twayne's World Authors Series } \\ \text { vgl. } & =\text { vergleiche, vergleichend } \\ \text { Vjschr. } & =\text { Vierteljahrsschrift } \\ \text { W.d. F. } & =\text { Wege der Forschung } \\ \text { Wiss. } & =\text { Wissenschaft, -lich } \\ \text { Zs. } & =\text { Zeitschrift } \\ \text { zit. } & =\text { zitiert }\end{array}$




\section{ABKüRZUNGEN ÖFTER GENANNTER LITERATUR}

BB

ER

Hegel S. W.

Heine

Herder S. W.

KAWSA

KFSA

KNA

LN

¿Das Nachleben

»Romantik «I

"Litkritik "
$=$ "Begriffsbestimmung der Romantik «, hrsg. H. Prang. W. d. F. 150. 1968.

$=$ »Die europäische Romantik«. E. Behler, H. Fauteck et al. 1973.

$=$ Jubiläumsausgabe, hrsg. H. Glockner. 20 Bde. $1927 \mathrm{f}$.

$=$ "Werke«, hrsg. E. Elster. 7 Bde. 1890, ${ }^{2} 1893$.

$=$ "Sämtliche Werke«, hrsg. B. Supphan. 33 Bde. 1877 f.

$=$ August Wilhelm Schlegel, »Ausgewählte Werke. Kritische Schriften und Briefe «, hrsg. E. Lohner. 6 Bde. 1962 f.

$=$ Friedrich Schlegel, »Historisch-Kritische Ausgabe«, hrsg. E. Behler, J. J. Anstetten u. H. Eichner. 18 Bde. 1958 f.

= Novalis' "Schriften «, hrsg. P. Kluckhohn u. R. Samuel. 4 Bde. 1928, ${ }^{2} 1960-75$.

$=$ Friedrich Schlegel, "Literary Notebooks « 1797-1801, hrsg. H. Eichner. Toronto 1957.

$=$ "Das Nachleben der Romantik in der modernen deutschen Literatur «, hrsg. W. Paulsen. 1969.

$=$ Die deutsche Literatur. Text u. Darstellung. Reclam 9629 f., hrsg. H. J. Schmitt. 1974.

$=\mathrm{R}$. Wellek, »Geschichte der Literaturkritik «. 1750-1830, übersetzt E. u. M. Lohner. 1959.

\section{Auflösung von Zeitschriftensigeln (NACH PMLA)}

$\begin{array}{ll}\mathrm{AL} & =\text { American Literature } \\ \mathrm{CL} & =\text { Comparative Literature } \\ \mathrm{CLS} & =\text { Comparative Literature Studies } \\ \mathrm{CollG} & =\text { Colloquia Germanica } \\ \mathrm{CWGV} & =\text { Chronik Wiener Goetheverein } \\ \mathrm{DN} & =\text { Delaware Notes } \\ \mathrm{DU} & =\text { Deutsch-Unterricht } \\ \mathrm{DVj} . & =\text { Deutsche Vierteljahrsschrift für Geistesgeschichte } \\ \mathrm{ELH} & =\text { J. Literaturwissenschaft } \\ \mathrm{ELN} & =\text { Engrnal of English Literary History } \\ \mathrm{EP} & =\text { Etudes Philosophiques }\end{array}$




$\begin{array}{ll}\text { FMod } & =\text { Filología Moderna } \\ \text { FR } & =\text { French Review } \\ \text { Goethejb. } & =\text { Jahrbuch der Goethe-Gesellschaft, Weimar } \\ \text { GQ } & =\text { German Quarterly } \\ \text { GRM } & =\text { Germanisch-Romanische Monatsschrift } \\ \text { JDSG } & =\text { Jahrbuch der deutschen Schiller-Gesellschaft } \\ \text { JEGP } & =\text { Journal of English and Germanic Philology } \\ \text { JFDH } & =\text { Jahrbuch des Freien Deutschen Hochstifts } \\ \text { JGG } & =\text { Jahrbuch der Grillparzer-Gesellschaft } \\ \text { JJPG } & =\text { Jahrbuch der Jean Paul-Gesellschaft } \\ \text { LI } & =\text { Lettere Italiane } \\ \text { LJGG } & =\text { Literaturwissenschaftliches Jahrbuch der Görres- } \\ & =\text { gesellschaft } \\ \text { MHG } & =\text { Mitteilungen der E. T. A. Hoffmann-Gesellschaft } \\ \text { MLQ } & =\text { Modern Language Quarterly } \\ \text { MLR } & =\text { Modern Language Review } \\ \text { NCFS } & =\text { Nineteenth Century French Studies } \\ \text { NDH } & =\text { Neue deutsche Hefte } \\ \text { NRs } & =\text { Neue Rundschau } \\ \text { PMLA } & =\text { Publications of the Modern Language Association } \\ \text { PQ } & =\text { Philological Quarterly } \\ \text { RF } & =\text { Romanische Forschungen } \\ \text { RFE } & =\text { Revista de Filología Española } \\ \text { RLC } & =\text { Revue de Littérature Comparée } \\ \text { RLI } & =\text { Rassegna della Letteratura Italiana } \\ \text { RLM } & =\text { Rivista di Letteratura Moderna } \\ \text { SFGG } & =\text { Spanische Forschungen der Görresgesellschaft } \\ \text { SGG } & =\text { Studia Germanica Gandensia } \\ \text { SIR } & =\text { Studies in Romanticism } \\ \text { SN } & =\text { Studia Neophilologica } \\ \text { ThS } & =\text { Theatre Survey } \\ \text { TSLL } & =\text { Texas) Studies in Literature and Language } \\ \text { TuK } & =\text { Text und Kritik } \\ \text { WB } & =\text { Weimarer Beiträge } \\ \text { WZPHP } & =\text { Wissenschaftl. Zs. der Pädagog. Hochschule Potsdam } \\ \text { WZUL } & =\text { Wiss. Zs. der Karl-Marx Univ. Leipzig } \\ \text { WZUR } & =\text { Wiss. Zs. der Univ. Rostock } \\ \text { YFS } & =\text { Yale French Studies } \\ \text { YWMLS } & =\text { Year's Work in Modern Language Studies } \\ \text { ZDP } & =\text { Zs. für deutsche Philologie } \\ \text { ZS } & =\text { Zs. für Slawistik } \\ & \end{array}$

